\title{
Reflets
}

Revue ontaroise d'intervention sociale et communautaire

\section{Prostitution : de la théorie de l'étiquetage à la pratique du vécu. La perception de cinq femmes qui font de la prostitution}

\section{Marie-Josée Jobin}

Volume 7, numéro 1, printemps 2001

Santé mentale et les défis de l'an 2001

URI : https://id.erudit.org/iderudit/026346ar

DOI : https://doi.org/10.7202/026346ar

Aller au sommaire du numéro

Éditeur(s)

Reflets : Revue ontaroise d'intervention sociale et communautaire

ISSN

1203-4576 (imprimé)

1712-8498 (numérique)

Découvrir la revue

Citer cet article

Jobin, M.-J. (2001). Prostitution : de la théorie de l'étiquetage à la pratique du vécu. La perception de cinq femmes qui font de la prostitution. Reflets, 7(1), 206-228. https://doi.org/10.7202/026346ar

Tous droits réservés (C) Reflets : Revue ontaroise d'intervention sociale et communautaire, 2001
Ce document est protégé par la loi sur le droit d'auteur. L'utilisation des services d'Érudit (y compris la reproduction) est assujettie à sa politique d'utilisation que vous pouvez consulter en ligne.

https://apropos.erudit.org/fr/usagers/politique-dutilisation/ 


\section{Prostitution : de la théorie de l'étiquetage à la pratique du vécu. La perception de cinq femmes qui font de la prostitution}

Marie Josée Jobin

Récipiendaire de la bourse RIFAS 2000,

École de service social, Université d'Ottawa ${ }^{1}$

Peu de chercheuses et de chercheurs se sont arrêtés à comprendre la prostitution féminine à partir du discours des femmes qui la vivent. C'est pourquoi nous pensons que notre étude qualitative, bien que limitée, entre autres, par le nombre restreint de femmes rencontrées, contribuera à faire ressortir une nouvelle dimension. La recherche est centrée sur la dynamique de la théorie de l'étiquetage dans le champ d'activité de la prostitution féminine. Notre but est de mieux comprendre comment les femmes qui font de la prostitution se perçoivent et comment elles perçoivent leur environnement.

Afin de mieux cerner notre objet de recherche, nous définissons d'abord de façon succincte la théorie de l'étiquetage pour ensuite entrer dans l'univers, l'imaginaire et la culture de rue des femmes qui font de la prostitution. Cela nous permet de dégager des pistes de recherche et d'intervention qui, nous l'espérons, favoriseront l'enrichissement de la réflexion et des interventions auprès de ces femmes.

Dans ce texte, nous avons choisi de retenir l'expression femmes qui font de la prostitution plutôt que femmes prostituées, afin de 
privilégier ce que font ou subissent ces femmes plutôt que ce qu'elles sont.

\section{La théorie de l'étiquetage}

Dans notre société, des groupes d'individus détenant un pouvoir politique, social ou économique dictent les normes sociales, ce qui a pour effet de cibler les individus qui ne respectent pas les normes prescrites. Un individu déviant est reconnu comme tel par «l'autre» parce qu'il adopte une conduite qui n'est pas conforme à la norme sociale. Les activités qui entourent la prostitution au Canada sont illégales et la prostitution est devenue par le fait même une forme de déviance, résultat d'un processus d'apprentissage conduisant à sa stigmatisation (Stanton 2000; Brock 1998; Kempadoo 1998; Bullough et Bullough 1998; Sharpe 1998; Queen 1997; Cusson 1992; Dorais 1988; Becker 1985; Goffman 1975). Le contrôle exercé sur des acteurs spécifiques tels que les femmes qui font de la prostitution sert à les étiqueter socialement. L'étiquette «prostituée un jour, prostituée toujours» colle bien à la vie des ces femmes.

Notre analyse privilégie la théorie de l'étiquetage parce qu'elle explique bien la déviance non criminelle qu'est la prostitution. Cette théorie permet également d'illustrer les dispositifs par lesquels un phénomène déviant peut se manifester dans une communauté sans déranger cette dernière, et ce, malgré les réactions punitives dont il est l'objet (Winterdyk 2000; Downes et Rock 1998; Meier et Geis 1997; Cusson 1992; Becker 1985; Eitzen et Timmer 1985; Goffman 1975).

L'œuvre de Becker intitulée Outsiders (1963; traduite en français en 1985) met en évidence la déviance non pas comme un trait spécifique à une personne ou à des actes, mais comme une caractéristique de l'interaction entre la société et cette personne ou ces actes. Becker écrit : 
La déviance n'est pas une qualité de l'acte commis, mais plutôt une conséquence de l'application, par les autres, de normes et de sanctions à un «transgresseur». Le déviant est celui auquel cette étiquette a été appliquée avec succès et le comportement déviant est celui auquel la collectivité attache cette étiquette (1985:33).

La déviance est donc le produit des interactions entre la personne et les normes sociales construites en fonction des valeurs dominantes de la société. La déviance est un processus arbitraire. Selon Cusson,

la déviance n'est pas une construction sociale tout à fait fantaisiste. La plupart du temps, les jugements créateurs de déviance sont des réactions à des actes qui victimisent autrui, qui perturbent sérieusement les proches du déviant ou qui affectent gravement le déviant lui-même. Il existe bel et bien une «donnée» antérieure au «construit» (1992: 397).

Becker considère que le processus de déviance veut attribuer un statut particulier à l'individu. Selon lui, la société crée elle-même la déviance en construisant des règles à briser et en étiquetant ceux qui contreviennent aux dits règlements comme déviants ou étrangers (outsiders). De fait, l'acte est déviant si et seulement si les autres le considèrent comme tel.

La société s'organise pour entretenir une certaine déviance. Lemert souligne que les effets négatifs de la non-conformité ou de la déviance sont neutralisés par un mécanisme de normalisation (Downes et Rock 1998; Gassin 1988). En ce qui concerne la prostitution, on a séparé les personnes impliquées dans la relation sexuelle et la reconnaissance du problème social est alors attribuée au seul comportement sexuel de la femme prostituée, comme si le phénomène dépendait exclusivement de celle-ci. Ce processus réaffirme la norme sexuelle admise par la collectivité. 


\section{Cadre et méthodologie de l'étude}

Parce qu'aucun cadre théorique ni aucune analyse n'équivalent à l'interprétation qu'en donnent elles-mêmes les principales intéressées, à savoir les femmes qui font de la prostitution, nous avons voulu, par le biais d'une étude exploratoire qualitative, laisser la parole à des personnes qui ont rarement la possibilité de s'exprimer et à des femmes que l'on étiquette comme déviantes selon les normes sexuées de la société. Notre collecte de données a eu lieu à l'hiver 2000, avec la participation de cinq femmes francophones de la région de Hull-Ottawa, à un entretien semidirigé d'une durée de soixante minutes. Chaque entretien a été une source riche d'informations de l'interprétation que fait une femme de la prostitution.

Du discours de ces femmes se dégagent leurs forces, leurs résistances, leurs acceptations, leurs concessions, leurs révoltes et leurs douleurs. Ce texte se veut un espace où les interprétations, les pensées et le vécu des femmes peuvent être rapportés sans balises préétablies. Par conséquent, leurs contradictions et leurs ambivalences sont considérées bien qu'elles ne soient pas supportées par un système. Les amplifications, les oublis sélectifs, les non-dit permettent à la fois de recueillir des informations riches et aussi de ne pas remettre en question les récits. Ceux-ci sont spontanés et, à certains moments, les femmes se distancent de leur vécu en se racontant à travers un autre corps. Ce qui nous intéresse, c'est de saisir comment les femmes vivent leur métier du sexe et le rôle de la société dans leur étiquetage comme déviantes.

L'histoire des femmes que nous avons rencontrées ressemble à celle de milliers de leurs consœurs de l'Antiquité à nos jours. Du discours de ces femmes se dessinent différentes trajectoires traversées par la peur, la honte, le courage et la détermination. Cinq thèmes circonscrivent cette trajectoire :le fait d'être étiquetée comme prostituée, les implications des pouvoirs, les différents acteurs des activités de la prostitution, les intervenants sociaux et la relativité du choix. 


\section{Le fait d'être étiquetée comme prostituée}

C'est à travers le processus de répression que les femmes s'identifient comme déviantes et elles revivent souvent le rejet dont elles ont déjà été victimes. $\mathrm{Si}$, avec le temps, les femmes se sont fait une carapace qui les protège en partie des regards et des commentaires des citoyens, des intervenants sociaux, de leurs proches, etc., leur réaction interne est toute autre : elles éprouvent de la honte. Devoir se définir comme prostituée implique que la femme rationalise les normes sexuelles de la société.

La femme "prostituée» fait de la déviance de la prostitution un mode de vie et organise son identité à partir de son comportement dit déviant. Becker mentionne que «ce sont des motifs socialement appris qui sont à l'origine de ces activités déviantes» (1985:53). Les femmes apprennent ainsi à socialiser selon la sous-culture de la rue. Becker stipule de plus que «le fait d'être pris et stigmatisé comme déviant a des conséquences importantes sur la participation ultérieure à la vie sociale et sur l'évolution de l'image de soi de l'individu» (1985 :54-55).La femme acquiert donc un nouveau statut, c'est une question de survie. Bien qu'elle se dise loin des regards des autres et des commentaires désagréables, elle est d'abord une personne et toute personne est atteinte par l'évaluation des autres.

Il y a du monde qui nous juge, ben moi asteure ça me passe 10 pieds par-dessus la tête. Tu veux me juger, pis me condamner, je m'en calisse. C'est ta perception et non la mienne. Ben moi la mienne, $j$ 'ai faite qu'estce que j'avais à faire. T'es pas content, that's your problem. Mais là je suis habituée, je sais où je m'en vais. (Claudine)

Pour certaines femmes, comme Marie et Josée, le fait d'être identifiées comme des femmes qui font de la prostitution les marquent profondément et elles viennent à adhérer à ce que les autres pensent d'elles, ce qui correspond au fondement de la théorie de l'étiquetage. 
Ben, il $y$ avait beaucoup de monde qui ne te respectait pas, qui criait : you are a prostitute. Je me sentais low low class. Je me sentais low class, bonne à rien, pis que j'étais une parasite envers le monde. Moi aussi je me le disais. Ça je pense que ça complète qu'est-ce que je suis. (Marie)

Il faut être faite dur. Il faut être rendu bas pour faire ça la prostitution. Il ne faut pas s'aimer. (Josée)

D'autres femmes, par ailleurs, rationalisent leur statut :

Pis après je me suis dit : pourquoi je m'en ferais, t'sais une fermme au foyer ben des fois, elle va donner du sexe à son mari quand il amène sa paye ou si elle veut avoir de quoi et elle va lui donner du sexe. T'sais ça l'arrive tous les jours, pour moi c'est une forme de prostitution. (Caroline)

Le problème n'est d'ailleurs pas la prostitution, mais bien la norme sociale qui est dictée par la société elle-même, celle-ci conditionnant les rapports inégaux de la prostitution (Brock 1998;Bullough et Bullough 1998; Sharpe 1998; Meier et Geis 1997). Il va sans dire que les appellations utilisées pour désigner les femmes qui font de la prostitution telles que fille de joie, pute, putain et prostituée consistent en des étiquettes exclusives au comportement sexuel des femmes. Celles-ci sont donc considérées déviantes par rapport à une norme sociale. Paradoxalement, aucun vocable n'étiquette les hommes-clients dans leur relation avec les femmes qui font de la prostitution (sauf peut-être l'appellation de johns) et les hommes ne sont perçus à aucun moment comme déviants. Par conséquent, aucune stigmatisation n'est attribuée aux hommes-clients, ce qui confirme leur comportement sexuel comme non déviant. Le double standard est éloquent.

Lorsque les femmes parlent d'elles-mêmes, elles disent d'abord et avant tout qu'elles sont des femmes, des personnes à part entière au même titre que les autres citoyennes. Les femmes restent avec un sentiment d'insécurité, elles ont une forte tendance à se sousestimer comme femme et à cause du rôle social qu'elles ont. De 
plus, lorsqu'elles sont arrêtées par les policiers, elles sont par le fait même stigmatisées, fichées, cataloguées et marginalisées, d'où le concept de la déviance secondaire défini par Lemert (Gassin 1988). Ce dernier distingue la déviance primaire de la déviance secondaire. Le déviant primaire est celui qui a fait un acte déviant quelconque sans être pris dans le processus de réaction sociale contre la déviance (Gassin 1988). La déviance primaire peut survenir dans diverses circonstances et sera peu significative pour l'individu étant donné que les autres ne l'ont pas étiqueté comme une mauvaise personne (Winterdyk 2000). La déviance secondaire est, par contre, identifiable lorsque le déviant primaire doit faire face aux réactions sociales des autres, par exemple, le contrôle social et la stigmatisation.

Lors des entretiens, les femmes ont soulevé le fait que les activités de la prostitution devraient être légalisées. Elles privilégient des endroits sécuritaires pour contrer la clandestinité et tout ce que ces activités impliquent. Il est à noter qu'en 1985, le Comité Fraser a servi de tampon vis-à-vis les pressions exercées par la population canadienne à l'égard de la prostitution de rue.Peu d'auteurs s'entendent quant aux mesures législatives à adopter : criminalisation, décriminalisation et réglementation (Stanton 2000; Meier et Geis 1997; Shaver 1988). Certains adhèrent à la décriminalisation de la prostitution en spécifiant que cette mesure se limite à la défense des femmes et non aux activités de la prostitution, tandis que d'autres conçoivent difficilement cette option. Les auteurs s'accordent toutefois pour dire que les solutions passent par des réformes économiques et sociales et non par le pénal.

Comme le mentionne Lacasse (1994), la répression ne visait pas et ne vise toujours pas l'institution de la prostitution, mais plutôt le contrôle des femmes. L'avocate Lippel stipule qu' «il y a une volonté politique de rendre la prostitution invisible, mais pas de l'interdire» (Baril 1989) parce que l'activité répond à des besoins.

La normalisation de la prostitution ne fait qu'excuser le comportement de l'homme étant donné que l'acte condamnable est exclusivement celui de la femme. Les valeurs dominantes de la société patriarcale encouragent la tolérance envers la femme et, 
la liberté sexuelle des hommes étant considéré comme un droit, les lois sont orientées vers le contrôle des femmes qui offrent le service sexuel (Stanton 2000). La législation de la prostitution sert, d'une part, à rassurer la collectivité et, d'autre part, à contraindre la femme qui fait de la prostitution à conserver son rôle dans la société.

L'étiquette est là pour fabriquer un petit nombre de déviants et ainsi assujettir et stigmatiser certains groupes tels que ceux des femmes qui font de la prostitution. Ce mode de discrimination et d'exclusion sociale se réalise par des lois, des mécanismes, des pratiques et des discours contribuant à produire des préjugés sociaux à l'égard des femmes qui font de la prostitution. En identifiant les femmes comme les «anormales» de l'activité de la prostitution, la collectivité construit une image sélective de la femme et se déculpabilise.

\section{Les implications des pouvoirs}

La prostitution soulève des rapports de pouvoir qui existent et qui répondent aux attentes de la société. La prostitution chez les femmes définit un double rapport de domination : celui des hommes sur les femmes et celui des nantis sur les démunis. Le pouvoir que donne l'argent à ceux qui le possèdent et le pouvoir politique ne sont pas à négliger. Dans le monde de la prostitution, le rapport s'exerce entre les clients, les policiers, les agents judiciaires, les souteneurs, etc. et les femmes. Stanton stipule que «[1]a prostitution demeure aussi florissante qu'avant et si plus de clients sont arrêtés aujourd'hui (on partait de zéro...), les prostituées restent d'abord dans le collimateur des policiers» (2000:25).

Les femmes qui font de la prostitution s'approprient un pouvoir lorsqu'elles se donnent le droit de décider des conditions de l'échange avec l'homme-client, notamment le droit de choisir le lieu, le montant, les «actes», le port du condom, le refus d'un homme-client, les parties du corps défendues, par exemple, la bouche. Les femmes font donc des choix qui modifient leur 
perception. Le pouvoir de négociation s'effectue en fonction des besoins respectifs des acteurs de l'activité de la prostitution. Les femmes qui font de la prostitution n'écartent pas, par ailleurs, le pouvoir des hommes-clients, mais veulent modifier la philosophie des acteurs de la prostitution.

Le pouvoir c'est moi, mais le client c'est quelqu'un que j'ai de besoin quand même. (Josée)

Les femmes qui font de la prostitution se permettent donc de dire qu'elles ne sont pas «entretenues» par un homme, c'est ainsi que les normes sexuées sociales sont secouées, c'est-à-dire que ces femmes montrent les choses différemment, sous un angle plus favorable à leur condition. Ainsi, elles considèrent cette activité comme un métier qui se définit par un échange de services entre l'homme-client et la femme qui fait de la prostitution. Comme dans tout métier, il y a des avantages (se sentir valorisée) et des inconvénients (loi du milieu). Les femmes utilisent des outils de travail, notamment le condom, la brosse et la pâte à dents, la drogue, parfois une arme, mais aucune des femmes rencontrées n'a fait référence à son corps pour définir son travail. Elles ne se perçoivent pas comme pourvoyeuses de services. La femme qui fait de la prostitution est d'abord une femme, une personne avec une personnalité et une identité, bien que nous lui accordons exclusivement celle de déviante.

Écoute ben ce n'est pas parce que tu me payes que tu vas m'appeler par des noms. (Caroline)

J'suis une personne humaine, pis que je m'aime là. (Marie)

Les femmes qui font de la prostitution et qui identifient cette activité comme un métier en arrivent à s'étiqueter elles-mêmes, mais bien que la réaction négative des autres les incite à se définir comme prostituées, elles n'abandonnent toutefois pas complètement l'image positive qu'elles ont d'elles-mêmes. À travers le regard des autres, les femmes se définissent, d'une part, comme déviantes selon les normes sexuelles décidées socialement et, d'autre part, comme une personne humaine qui a des besoins à satisfaire. Il importe de 
souligner que les femmes ne naissent pas prostituées, elles le deviennent plutôt à cause de certains éléments individuels certes, mais davantage sociaux (Bullough et Bullough 1998; Sharpe 1998; Meier et Geis 1997; Cusson 1992; Dorais 1988; Becker 1985). Réagissant à la stigmatisation de leur rôle social, les femmes s'organisent pour négocier les conditions de l'échange avec l'homme-client. De cette façon, les femmes contrôlent en partie leur vie.

Par ailleurs, la femme qui fait de la prostitution est d'abord une femme, une personne à part entière, soit une mère, une amie, une amante. Le respect de sa vie privée est primordial parce qu'en dehors de son travail comme prostituée, la femme a d'autres occupations. Certaines femmes rencontrées pour la recherche ont partagé avec leurs proches leur travail comme prostituée. Elles n'exigent pas d'eux qu'ils soient en accord avec leur choix, bien que ce que pensent les autres et particulièrement les proches les influencent dans leur choix de vie comme prostituée. Mais un sentiment de culpabilité et de honte peut amener les femmes à cacher leur travail. Certaines femmes n'ont plus de contact avec leur famille, d'autres entretiennent une relation avec un frère, une sœur, leur mère. Parce que la société n'accepte pas le métier de prostituée, la femme doit vivre dans la clandestinité et la marginalité, ce qui ne facilite pas le respect des autres à son égard. Les femmes qui font de la prostitution sont condamnées par leur statut de prostituée.

Les femmes partagent des projets qu'elles veulent réaliser ultérieurement et un même message se dégage : elles veulent s'accorder du temps et un espace pour elles-mêmes parce qu'elles sont importantes. Leurs besoins se mesurent différemment aujourd'hui d'il y a quelques années, notamment comme le besoin de consommer a diminué il y a également baisse dans la nécessité de faire de la prostitution.Les objectifs se modifient : connaitre ses enfants, avoir une vie amoureuse et réorienter sa carrière deviennent des buts.

Le désir d'«aider» les jeunes femmes qui commencent à faire de la prostitution est un autre élément du discours des femmes. Deux types d' «aide» se distinguent, dont un premier visant surtout à répondre à des besoins d'ordre matériel : 
Moi, j'ai toujours voulu être travailleuse de rue. Oni, parce que je sais que je suis capable, $j$ 'suis dans ce milieu là, j'étais dans ce milieu là. J'serais capable de passer des seringues, des condoms, y a rien là. Mais jusqu'à aller dans les piqueries, je serais pu capable. (Claudine)

La deuxième type d'aide axe davantage l'intervention vers les jeunes femmes elles-mêmes qui font de la prostitution dans le but de les sortir de cette activité. Les jeunes femmes sont souvent sous l'emprise d'un souteneur qui leur promet la protection en échange d'une partie de leur gain. C'est avec émotion que Caroline parle d'une jeune fille sous l'emprise d'un souteneur :

Fait que là,j'vas voir la petite : pis pourquoi tu pleures, pis elle pleurait. Pis là, elle commence à me conter qu'elle venait de Vancouver pis là le gars (souteneur) l'avait amenée là. Pis il était supposé de la gâter. Ils les gâtent au commencement. Je l'ai amenée chez nous, j'ai appelé un client que je connaissais, $j$ 'avais un sugar daddy. J'ai dit :ça prend $1000 \$$ tout de suite. Il a dit pourquoi? J'ai dit: j'ai une petite fille, je veux la mettre sur l'avion pour l'envoyer chez eux. J'espère que ça bien été. T'sais elle peut-être, que ça été une leçon de vie pour elle. T'sais là, elle est grande. (Caroline)

Ces femmes reconnaissent également leurs limites potentielles quant à leur rôle d'aidante; tout ce qu'implique le retour dans le milieu de la drogue et les émotions d'un vécu sont notamment pris en considération. Certaines femmes ressentent le besoin de s'accorder du temps pour elles; jusqu'à maintenant elles ont donné aux autres, soit aux hommes-clients, à un frère, à une sœur, à un conjoint et par le fait même se sont oubliées comme personne. Les femmes vont toutefois jusqu'à dire qu'elles se sentent égoïstes, ce qui nous permet de croire qu'elles ont bien intégré l'image sociale. En identifiant les femmes comme les «anormales» de l'activité de la prostitution, la collectivité construit une image sélective de la femme et se déculpabilise. 


\section{Les différents acteurs des activités de la prostitution}

\section{Les clients}

Les activités de la prostitution exigent au moins deux acteurs, soit les hommes-clients et les femmes qui font de la prostitution. Sans client, pas de prostitution. C'est une certitude que l'on finit par omettre. Étant donné que les femmes qui font de la prostitution considèrent cette activité comme un échange de services, le respect mutuel entre l'homme-client et la femme est donc une condition favorable à cet échange. Le respect se définit de plusieurs façons, par exemple, lorsque les femmes exigent le port du condom, elles pensent à se protéger autant elles-même que le client, mais de surcrôt, certaines considèrent aussi la partenaire de ce dernier et le fotus potentiel de ce couple. Toutefois la situation peut être toute autre. Si, d'une part, le besoin de consommer est urgent, la femme peut prendre des risques afin de satisfaire à son besoin. D'autre part, si l'homme client donne plus d'argent pour ne pas utiliser le condom, la femme peut accepter cet arrangement, ce qui lui permet de «faire» un client de moins.

Les femmes respectent aussi les hommes-clients quant aux genres de demandes si celles-ci ne les mettent pas en danger. Les femmes s'entendent pour dire qu'elles jouent différents rôles avec l'homme-client et que cela nécessite de l'imagination et de la flexibilité. Une autre forme de respect se dessine dans le discours des femmes, à savoir qu'elles ne généralisent pas les comportements des hommes-clients.

Le manque de respect est aussi un élément dans les relations hommes-clients et femmes qui exercent la prostitution, et se manifeste dans la violence subie par ces dernières. Par ailleurs, la coopération des hommes-clients avec la femme est souvent déterminante lorsqu'ils sont en présence des autorités.

En conclusion, bien que les femmes détiennent certains éléments de pouvoir, il ne faut cependant pas omettre le pouvoir de celui (homme-client) qui a l'argent. La négociation se fait entre les besoins de ce dernier et ceux de la femme qui fait de la prostitution. 


\section{Les souteneurs}

Le rapport entretenu entre les femmes expérimentées et les souteneurs est quasi inexistant. Ces femmes sont unanimes pour mentionner que les souteneurs ne leur apportent aucune sécurité et elles déconstruisent ainsi le mythe si souvent véhiculé à propos de ces acteurs dans l'activité de la prostitution. Les femmes ont ainsi développé entre elles un système de jumelage. Elles réussissent à se faire confiance et elles partagent la même perception quant aux limites de la protection sur la rue. D'emblée, les femmes expérimentées ne se font pas imposer la présence d'un souteneur dans leur activité, alors que celui-ci promet aux plus jeunes femmes sa protection en échange d'une somme financière.

\section{Les paires}

Les interactions entre les paires, c'est-à-dire les femmes qui font de la prostitution, favorisent la première intégration des règles du milieu (Sharpe 1998). Lors de cet apprentissage, toutes les femmes vivent une étape commune et définitive : l'intériorisation d'un nouveau rôle, celui d'être une prostituée. Ce rôle est personnalisé pour chacune des femmes et il favorise l'appropriation des stéréotypes qui la définiront face aux autres.

Afin de survivre, les femmes s'entraident sur divers plans : l'uniformité des prix, l'identification des mauvais clients et la surveillance mutuelle. Elles sont continuellement en compétition pour assurer leur survie mais elles doivent s'en tenir aux tarifs réguliers à défaut d'être réprimandées par leurs paires. Les activités de la prostitution impliquent autant la complicité que la rivalité entre les paires. Les rôles déterminent les comportements des femmes selon les activités faites et, dépendamment des acteurs sociaux, les femmes se font confirmer leur étiquette.

\section{Les acteurs sociaux}

La femme qui fait de la prostitution est confrontée à différents contrôles, notamment le regard des citoyens et des citoyennes (les femmes et les enfants ont davantage de poids pour susciter la 
honte et le désarroi) et l'arrestation de la police qui les culpabilise et les condamne officiellement. Suite à cette marque publique, les femmes s'identifient comme des personnes déviantes des normes sexuelles sociales, un processus qui les stigmatise.

Avoir peur des clients et des policiers, être obligées de se cacher et de continuellement surveiller ses arrières correspondent à des risques du métier avec lesquels les femmes doivent apprendre à composer si elles veulent "vivre». Les femmes reconnaissent de plus le pouvoir des policiers à leur égard. C'est à travers cette répression que la femme prostituée s'identifie comme différente, marginale. Faut-il ajouter que toute répression aux activités de la prostitution n'a eu aucune efficacité; la prostitution existe et existera toujours, par conséquent il est inutile de culpabiliser les femmes (Stanton 2000; Bullough et Bullough 1998; Sharpe 1998; Meier et Geis 1997; Nadeau 1993; Shaver 1988; Bournival 1987).

T'sais y (policiers) travaillent pas avec leurs attitudes, t'sais y écourent pas les filles. T'sais y tapent pas sur les nerfs. Comme y en a une couple, j'leur twisterais la tête de ses épaules.Y te suivent partout, y t'écourent, $y$ t'harcèlent. (Claudine)

Les femmes sont constamment sur le qui-vive et doivent planifier des actions stratégiques et développer ainsi des mécanismes de survie. Elles vivent un jour à la fois. De fait, les femmes rapportent leur rythme de vie dans un discours où s'entremêlent la fierté de vivre et la «dure» réalité du milieu. Par ailleurs, elles côtoient également d'autres intervenants sociaux, notamment les travailleurs de rue. Leurs relations avec ces derniers sont qualifiées d'humaines et répondent à différents besoins tels que d'échanger à propos d'une situation, de manger, de dormir, de se protéger lors des contacts sexuels et lors de consommation (échange de seringues).

Les femmes qui font de la prostitution ont besoin d'être sécurisées dans leur prise de décisions, car elles vivent continuellement dans l'insécurité. Elles apprécient par conséquent la relation avec les travailleurs de rue, qui est possible grâce à l'accessibilité et à la disponibilité de ceux-ci. Les femmes ont besoin, comme toutes autres personnes, d'être respectées dans leur cheminement 
de vie, d'être écoutées et validées et, à cet effet, les travailleurs de rue sont des personnes ressources précieuses. De surcroît, un lien de confiance se tisse entre les travailleurs de rue et les femmes, car leur relation n'est pas menaçante pour quiconque.

Ils (travailleurs de rue) sont ben utiles pour jaser, pour avoir des condoms. Eux autres ils apparaissent pratiquement n'importe où, n'importe quand, toujours quand tu en as de besoin comme ça, pis ça c'est bon. Ils vont être ouverts à n'importe quoi tu vas leur dire, ils vont t'écouter, pis souvent ils vont dire pis so what comme tu sais qu'est-ce qui peut t'arriver. Ils dédramatisent les choses. T'sais eux autres écoutent, qu'est-ce que le citoyen straight ne ferait pas parce que ça scandaliserait certaines personnes. Pis tu as agi comme ça, pis eux autres comme ils te disent que ça fait partie de la game. T'sais que les travailleurs de rue tu peux leur raconter des affaires parce qu'ils s'en feront pas avec qu'est-ce que tu dis. (Josée)

Parce que la combinaison de la pauvreté, de la dépendance et de la stigmatisation maintient certaines femmes dans la prostitution, nous pouvons comprendre pourquoi elles poursuivent leurs activités et qu'elles acceptent des compromis, par exemple, de baisser les prix pour un client afin de satisfaire un besoin de consommation même s'ils ne répondent pas aux attentes du milieu

\section{La relativité du choix}

La prostitution est un processus d'apprentissage; on ne nait pas prostituée, on le devient suite à des interactions dans un contexte culturel et des réalités sociales particulières. L'entrée dans le monde de la prostitution se réalise par des étapes d'intégration successives : la femme adhère à une image négative d'elle-même; elle assimile le comportement associé à cette image qui est confirmée par un étiquetage informel; elle s'intègre à la sous-culture et développe ainsi un caractère déviant. Enfin, elle s'identifie comme prostituée 
avec l'étiquette formelle (Bullough et Bullough 1998; Meier et Geis 1997; Dorais 1988; Becker 1985; Goffman 1975). La femme suit souvent un processus de victimisation qui a débuté lors de l'enfance avec des expériences violentes, par exemple des abus, et qui se perpétue avec la prostitution. Elle arrive à s'auto-détruire à cause des réactions négatives des autres à son égard. Étiquetées et stéréotypées, les femmes qui pratiquent la prostitution ne peuvent rester indifférentes au jugement de leur entourage, la perception des choses et des individus étant issue des interactions de la vie.

Une fois la prostitution vue comme élément de solution pour les femmes, il leur devient difficile de voir d'autres éléments de solutions pour plusieurs raisons, notamment l'intégration de l'image et de la vie de prostituée. Le discours des femmes qui ont décidé d'opter pour la rue (ou serait-il plus juste de dire que c'est la prostitution qui les a choisies) s'inscrit dans une dynamique individuelle, mais surtout sociale et de processus - besoin d'aider un frère, une sœur, besoin de vengeance, besoin de consommer, besoin d'argent rapidement, besoin d'échapper à quelqu'un ou à une situation, besoin d'être quelqu'une, etc.

J'ai commencé à l'âge de 14 ans, j'avais pas le choix. Ma mère nous a délaissé, pis c'est ça, j'ai été obligée de prendre soin de ma petite soeur, pis mon petit frère qui avaient besoin de sous pour aller à l'école, pis tout ça. J'ai dit :fuck it, je m'en vais travailler. (Claudine)

D'abord retenue comme solution pour combler un besoin à court terme, la prostitution devient un mode de vie pour les femmes qui ont été identifiées par les autres comme prostituées et s'identifient ensuite elles-mêmes comme telles.

Ça ne me dérange pas, mais pas pantoute. Avant, ça me dérangeait mais c'est comme tu veux m'identifier de même, c'est ton hostie de problème. Ça c'est une fille de rue, so what ça dérange quoi dans ta vie. Ça l'air à déranger beaucoup en hostie, c'est ça que je leur dis. (Claudine) 
Avant ça me touchait beaucoup, chu ben émotionnelle. (Caroline)

Nous nous interrogeons sur le sens des dires de Caroline lorsqu'elle décrit son enfance comme étant heureuse et que quelques minutes après, elle se précipite pour raconter le viol par son cousin qu'elle a subi dans sa jeunesse. De fait, Caroline dit avoir commencé la prostitution dans le but de faire payer aux hommes le comportement abusif qu'elle a subi lors de son enfance. D'autres femmes penseront par ailleurs que leur corps, suite à des abus, ne mérite rien. Évidemment ce ne sont pas toutes ces femmes qui ont subi de l'inceste et elles n'expliquent pas nécessairement leur prostitution par l'abus qu'elles ont subi dans leur enfance.

Dans le discours des femmes, la prostitution est prise comme un moyen de subsistance, d'évasion, et le besoin de consommer est omniprésent. La prostitution, la drogue, les maladies transmises sexuellement et la violence ainsi que toutes les implications sousjacentes constituent la sous-culture du milieu des femmes qui apprennent les règles du jeu. La notion et la valeur de l'argent sont également des aspects particuliers discutés par les femmes qui disent que c'est tellement payant.

Selon leurs schèmes de références, les femmes s'entendent pour dire que l'argent des activités de la prostitution leur permet de satisfaire d'abord à un besoin de consommation car la drogue est nécessaire. Il importe de comprendre que pour être, il faut d'abord consommer de la drogue, se construire une identité et donc se donner du pouvoir. Par conséquent, il est indispensable d'avoir pour ensuite être. L'argent justifie également la présence des femmes sur la rue et leur existence.

Moi, je le fais parce que c'est payant, moi quand je veux avoir de l'argent tout de suite, je le fais. C'est ça qui a, tu tombes vite dans l'engrenage de faire de l'argent facile. (Caroline)

Pis à part de ça, je ne respecte pas l'argent autant quand je faisais ça (la prostitution) parce que ça allait toute dans drogue. (Marie) 
Mais d'autres femmes comme Claudine expriment qu'elles ont besoin d'affection et de tendresse et d'ailleurs c'est ce que recherchent aussi certains hommes-clients. Certaines femmes se disent enrichies et plus indulgentes par leur parcours dans la prostitution. La prostitution est sans équivoque une expérience marquante.

J'suis contente d'avoir toute faite ça parce que j'ai l'expérience qu'une citoyenne en guillemet n'a pas. Moi avec tout ça, j'ai appris beaucoup. Je l'apprécie beaucoup d'avoir passé par ce chemin là. (Claudine)

Mais c'est un engrenage, tu viens que tu t'aimes plus hen. Pis tu t'en fous. (Caroline)

La consommation de drogues implique une double contrainte, soit la dépendance de la drogue et l'obligation de la dette. Les femmes disent majoritairement qu'elles font de la prostitution parce qu'elles ont besoin de consommer des drogues pour toutes sortes de raisons telles que s'évader et oublier le mal fait à leur âme et à leur corps. Néanmoins, une participante mentionne qu'elle n'absorbe pas de drogues intraveineuses afin de réduire les risques. Adopter de tels comportements signifie qu'elles pensent à elles. Serait-il juste de se questionner à propos du comportement des hommes-clients? Sont-ils aussi consciencieux quant à leurs responsabilités dans l'activité de la prostitution?

J'avais fait une échange de seringues, pis j'aurais pas dû faire ça, c'était stupide, c'était vraiment stupide de ma part. Ben c'est fait, c'est fini. Moi je vis avec that's it. Ça m'empêche pas de vivre. (Claudine)

Pareil quand je faisais la drogue, je le disais à tout le monde que j'étais positive pour pas qui touche mes affaires. (Marie)

De fait, seules les femmes, depuis toujours, sont ciblées comme responsables des conditions de santé (Bullough et Bullough 1998; Sharpe 1998; Meier et Geis 1997; Nadeau 1993; Shaver 1988; 
Bournival 1987). Cela illustre une fois de plus comment une société détermine ses limites sociales en fonction du sexe des acteurs. Que ce processus soit admis démontre bien la tolérance qu'une société peut avoir à l'égard de l'activité de la prostitution, et surtout à l'égard d'un acteur, soit l'homme-client.

L'activité de la prostitution implique également la violence à l'égard des femmes, soit par les clients, les autorités, les autres femmes du milieu, les voisins, les monsieurs et madames tout le monde qui portent des jugements et la clandestinité des activités. Il importe de mentionner que les autres identifient les femmes comme déviantes à partir d'un schème de références sexuel normalisé socialement. C'est donc dire que ces femmes sont stigmatisées à partir des valeurs véhiculées dans la société et, ce qui importe davantage, c'est que celles-là sont déterminées en fonction des critères de groupes de personnes autres que les femmes qui font de la prostitution. La violence est omniprésente et la peur précède tout, soit les contacts avec les policiers, les clients et les autres femmes. Malgré toutes les contraintes qu'impliquent les activités de la prostitution, peut-être même à cause de ces contraintes, les femmes disent prendre un jour à la fois, de sorte qu'elles tentent de composer avec les circonstances et d'être conscientes des risques. La prostitution représente cependant un moindre mal pour les femmes, comparé aux autres choix, soit de manquer de drogues, d'abandonner son frère et sa sœur ou d'être pauvre.

Par ailleurs, à travers les activités de la prostitution, peut-être à cause d'elles, les femmes développent un pouvoir par rapport à leurs exigences pour le service rendu. Cependant, la répression cible spécifiquement une actrice des activités, soit la femme, et celle-ci apprend à s'identifier comme déviante sexuelle.

\section{Conclusion}

En somme, par la théorie de l'étiquetage, il est possible de démontrer que les femmes qui font de la prostitution ont appris leur rôle social et que celui-là les stigmatise. D'emblée, la stabilité 
sociale n'est pas remise en question par les activités de la prostitution, parce que la population les tolère, étant donné que les lois donnent l'impression de surveiller ces activités. Les femmes commencent la prostitution souvent pour des fins personnelles, mais poursuivent parce qu'elle permet de répondre à des besoins surtout de consommation (d'ordre financier) et une fois qu'elles sont identifiées comme déviantes, elles s'auto-étiquetent et en viennent à intégrer leur nouveau rôle social. Par conséquent, la société joue un rôle de premier ordre dans la stigmatisation des femmes en fonction des transgressions des comportements blâmables.

Les femmes qui font de la prostitution privilégient certaines conditions dans l'échange avec les hommes-clients, ce qui leur permet de se respecter comme personnes; toutefois la violence et la consommation sont des impératifs de leurs activités. Il importe de souligner que ces activités, comme d'autres, requièrent une période d'apprentissage, mais elles se font dans la clandestinité. Par ailleurs, certaines femmes se valorisent en disant être capables d'aider les jeunes femmes dans leurs activités. Elles expriment également le besoin de penser d'abord à elles, même si l'engrenage n'est pas simple à défaire.

La répression des citoyens à l'égard de ces femmes enclenche chez elles un processus d'autoétiquetage de déviantes sociales. Certaines femmes rationalisent leur statut, tandis que d'autres restent imprégnées de l'exclusion sociale. Pour survivre, les femmes doivent par conséquent endosser ce nouveau rôle que la société leur a défini. Malgré la croyance populaire, ces femmes sont d'abord des êtres humains et tout individu a droit au respect.

Les activités de la prostitution impliquent certains acteurs, notamment les femmes, les hommes clients, les souteneurs, les paires et les intervenants sociaux. Il importe de mentionner que ces activités nécessitent au moins deux acteurs et que ceux-ci sont traités socialement différemment selon leur sexe et qu'évidemment, le pouvoir de l'argent n'est pas à exclure. Par ailleurs, la culture de rue suppose certaines règles formelles, mais davantage informelles, entre les acteurs et spécialement entre les femmes. La coopération entre elles leur donne certaines balises de sécurité. 
Ce qui marque les femmes est sans aucun doute le jugement des autres et encore davantage l'arrestation par les policiers. Suite à cette étiquette formelle, les femmes s'identifient comme déviantes et intègrent le comportement de prostituée. Il importe de spécifier que la répression n'arrête pas les activités de la prostitution, elle sert plutôt à rassurer l'opinion publique et surtout à culpabiliser les femmes.

Cependant, il est trop facile de seulement dire aux femmes d'arrêter de faire de la prostitution si, en contrepartie, nous n'avons pas d'autres alternatives à leur offrir. Dans le même ordre d'idées, des conditions de travail plus saines et plus sécuritaires pourraient améliorer les conditions de vie des femmes. À cet effet, Stella un groupe de travailleuses du sexe et d'anciennes travailleuses du sexe - milite pour l'obtention de conditions de travail décentes.

Pour que le processus d'intervention auprès des femmes se réalise, il importe d'abord de les considérer comme des personnes ayant besoin d'aide et ayant également un potentiel.Trop souvent, les femmes ont été perçues comme des criminelles parce que certaines activités inhérentes à la prostitution s'inscrivent dans le Code criminel. Cependant, la prostitution n'est pas illégale, c'est plutôt le fait de solliciter quelqu'un sur la place publique qui constitue une infraction à la loi. Il est certain que les mesures actuelles à l'égard de la prostitution ne font que déplacer le problème et isolent surtout ces femmes.

L'appui apporté par les intervenants communautaires est considérable quant à l'aide donnée aux femmes. Les intervenants accordent une présence et une disponibilité, notamment en écoutant les femmes, en les valorisant et en les accompagnant dans leur cheminement personnel.

Étant donné que les hommes-clients sont des acteurs nécessaires au même titre que les femmes qui font de la prostitution, il s'avère important de sensibiliser tous les hommes à l'abus de ces femmes et aux conséquences de cet abus. Il est évident qu'une socialisation non sexuée aiderait autant les femmes que les hommes à identifier autrement les comportements sexuels acceptables. 
Que dire de la responsabilité sociale qui incombe à tous? Parce que la société crée la déviance des femmes qui font de la prostitution et non celle des hommes-clients, elle se doit également de jouer son rôle pour une éventuelle resocialisation non sexuée.

\section{Bibliographie}

BARIL, Daniel (1989), «La prostitution qui ne fait plus le trottoir», Justice, XI(2), 28-29.

BECKER, Howard S. (1985 [1963]), Outsiders : Études de sociologie de la déviance, trad. de l'américain par J.-P. Briand et J.-M. Chapoule, Paris, Éditions A.-M. Métailié.

BERCHAT, Maurice (1979), "Comment les prostituées se voient-elles?», Revue internationale de criminologie et de police technique, 32(1), 62-66.

BIBEAU, Gilles et Marc PERREAULT (1995), Dérives montréalaises, Québec, Boréal.

BOURNIVAL, Marie-Thérèse (1987), «La nouvelle loi du plus vieux métier... un an après», Justice, IX(2), 38-43.

BROCK R., Deborah (1998), Making Work, Making Trouble: Prostitution as a Social Problem, Toronto, University of Toronto Press.

BUllOUGH, Bonnie et Vern L. BUlLOUGH (1998), «Female Prostitution: Current Research and Changing Interpretations», dans James E. Elias, Vern L. Bullough, Veronica Elias et Gwen Brewer (sous la dir. de), Prostitution: On Whores, Hustlers and Johns, New York, Prometheus Books, 23-44.

Code criminel de poche (1997), Ontario Carswell.

COMITÉ FRASER (1985), La pornographie et la prostitution au Canada. Rapport du Comité spécial d'étude de la pornographie et de la prostitution, Ottawa, Approvisionnement et Services Canada, vol. 2.

CUSSON, Maurice (1992), «Déviance», dans Raymond Boudon (sous la dir. de), Traité de sociologie, Paris, Presses universitaire de France, 389-422.

DORAIS, Michel (1988), «La politique de la marginalisation sexuelle ou l'identité déviante. Le cas de l'homosexualité masculine et de la prostitution féminine», Le travailleur social, 56(2), 54-59.

DOWNES, David et Paul ROCK (1998), Understanding Deviance: A Guide to the Sociology of Crime and Rule Breaking, $3^{\text {rd }}$ ed., Oxford, Oxford University Press.

EITZEN, Stanley D. et Doug A. TIMMER (1985), «Theory of Crime and Criminal Justice», dans Criminology, Canada, John Wiley \& Sons Inc., 10-56.

GASSIN, Raymond (1988), Criminologie, France, Dulloz.

GOFFMAN, Erving (1975 [1963]), Stigmate, Paris, Éditions de minuit.

JONES, Stephen (1998), «Interactionism and Phenomenology», dans Criminology, Toronto, Butterworths, 177-201.

KEMPADOO, Kamala (1998), «Globalizing Sex Workers' Rights», dans de Hamala Kempadoo et Jo Doezema (sous la dir. de), Global Sex Workers, New York/London, Routledge, 1-28.

LACASSE, Danielle (1994), La prostitution féminine à Montréal, 1945-1970, Montréal, Boréal.

MEIER, Robert et Gilbert GEIS (1997), Victimless Crime? Los Angeles, California, Roxbury Publishing Company. 
NADEAU, Carole-Line (1993), «Prostitution : un choix par défaut», 15(1), 7-10.

QUEEN, Carol (1997), «Sex Radical Politics, Sex-Positive Feminist Thought, and Whore Stigma», dans Jills Nagle (sous la dir. de), Whores and other Feminists, New York/London, Routledge, 125135.

SHARPE, Karen (1998), Red Light, Blue Light: Prostitutes, Punters and the Police, Aldershot \& Brookfield USA \& Singapore \& Sydney, Ashgate.

SHAVER, Frances M. (1988), «A critique of the feminist charges against prostitution», Atlantis, 14(1), 82-88.

STANTON, Danielle (2000), «Un crime? Prostitution», La Gazette des femmes, 22(1), 18-25.

WINTERDYK, John A. (2000), «Labelling theory», dans Canadian Criminology, Scarborough, PrenticeHall Canada Career et Technology, 238-246.

Note

1. Octroyée depuis 1998 à une étudiante de l'École de service social de l'Université d'Ottawa, la bourse du Regroupement des intervenantes et des intervenants contre les agressions sexuelles (RIFAS) vise à souligner l'excellence d'un mémoire de maitrise portant sur la violence. Le mémoire de Mme Jobin a été dirigé par le professeur Gilles Renault. 